\title{
Presumed Orbital Infarction Syndrome after Functional Endoscopic Sinus Surgery (FESS)
}

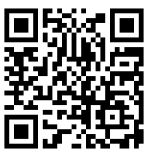

\author{
Randa MA El Mofty ${ }^{1}$, Rehab R Kassem*2, Farouk Hassan ${ }^{3}$, Ghada Ismail Gawdat ${ }^{4}$ and Rania A Ahmed $^{5}$ \\ ${ }^{1}$ Lecturer of Ophthalmology, Department of Ophthalmology \\ 2,4,5 Professor of Ophthalmology, Department of Ophthalmology
}

${ }^{3}$ Lecturer of Interventional Radiology, Department of Radialogy

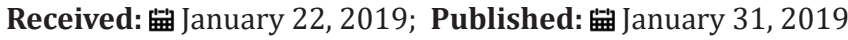

*Corresponding author: Rehab R Kassem, Ophthalmology department, Kasr Alainy hospital, Cairo, Egypt

\begin{abstract}
Purpose: This case report presents the first combination of optic nerve injury, central retinal artery occlusion, together with superior division oculomotor nerve palsy following functional endoscopic sinus surgery (FESS). It also presents the first case of intra orbital injury contralateral to the side of FESS.

Methods: A 23-year-old female was referred to the ophthalmic department due to development of a left dilated fixed pupil during right FESS. Ophthalmic examination revealed left complete ptosis, exotropia and hypotropia, with limited motility in all directions of gaze except abduction and depression. Vision was right 20/20 and left no perception of light. Left pupil was mid-dilated with loss of direct but preserved consensual reaction (afferent pupillary defect with intact efferent reaction). Fundus examination revealed a left central retinal artery occlusion. Right eye examination revealed normal anterior and posterior segments. CT scan and MRI revealed defects of the upper nasal septum and left lamina papyracea. The left medial rectus muscle and optic nerve were entrapped within the lamina papyracea defect. The left extraocular muscles were swollen. There was rarefaction of the bony ethmoidal septa. The right orbit was normal.
\end{abstract}

Results: Left optic nerve injury, central retinal artery occlusion, medial rectus entrapment, and palsy of the superior division of the oculomotor nerve were presumed. Orbital surgery, 7 months later, although successfully freed the entrapped medial rectus and closed the defect, yet no improvement in ocular motility or alignment ensued.

Conclusion: Care should be taken during FESS to avoid intraorbital injury. More care is essential in case of pre-existing septal defect or rarefaction of ethmoid sinus to avoid contralateral orbital complications.

Keywords: FESS Orbital Complications; Central Retinal Artery Occlusion; Optic Nerve Injury; Third Nerve Palsy; Lamina Papyracea; Muscle Entrapment

Abbreviations: FESS: Functional Endoscopic Sinus Surgery; no PL: no Perception of Light; CRAO: Central Retinal Artery Occlusion; SR: Superior Rectus; MR: Medial Rectus; IO: Inferior Oblique; SO: Superior Oblique; LR: Lateral Rectus; IR: Inferior Rectus; LPS: Levator Palpebrae Superioris

\section{Introduction}

Functional Endoscopic Sinus Surgery (FESS) is a surgical treatment of sinusitis and nasal polyps, using nasal endoscopes. The use of powered cutting instruments has been a recent advance in endoscopic sinus surgery, but it has also been implicated in rapid, irreversible, and devastating complications. The intimate relation between the paranasal sinuses and the orbit places the orbital contents at risk of injury during sinus surgery, especially surgery of the ethmoid sinuses. The orbit, the extra-ocular muscles, the optic nerve and the lacrimal drainage system can be damaged during FESS. The risk of injury is correlated to the anatomical variations, the history of previous surgery, the extent and the gravity of the disease and the skill of the surgeon [1].

\section{Case Description}

A 23-year-old female was referred to the ophthalmic department of Cairo University hospital immediately after undergoing a third FESS of the ethmoid sinus under general anesthesia, to treat nasal polyps and pansinusitis, due to suspected ophthalmic complications during the procedure. The patient had undergone 2 previous failed surgical procedures to treat nasal polyps. During 
the third right FESS, accidental injury to the left lamina papyracea occurred. The surgeon, then, noticed a left dilated fixed pupil. Ophthalmic examination revealed left complete ptosis. The left eye was exotropic and hypotropic with limited motility in all directions of gaze except abduction and depression. Corneal sensation was intact. Vision was no Perception of Light (no PL) in OS and 20/20 in OD with correction. Left pupil was mid-dilated with loss of direct but preserved consensual reaction (afferent pupillary defect with intact efferent reaction). Fundus showed a picture of left Central Retinal Artery Occlusion (CRAO) with pale elevated optic disc, pale retina and a cherry red spot. Right eye examination revealed normal anterior and posterior segments.

Systemic carbonic anhydrase inhibitor and Streptokinase were prescribed. The next day, the patient developed lid swelling, severe ecchymosis, subconjunctival hemorrhage, and mild proptosis in OS, together with mild ecchymosis in OD. On the third postoperative day, the patient developed corneal abrasions due to lower eyelid entropion. This was successfully treated with antibiotic eye ointment and eversion of lid when it rolled in. Closer examination of extraocular motility, one week later, revealed left limited elevation and adduction, suggestive of left Superior Rectus (SR) and Medial Rectus (MR) limited motility. There was left preserved extorsion on attempted elevation on adduction and intorsion on attempted depression on adduction, suggestive of intact left Inferior Oblique (IO) and Superior Oblique (SO), respectively, despite their failure to elevate or depress the eye being in an abducted position. There was left preserved abduction and depression, suggestive of normal left Lateral Rectus (LR) and Inferior Rectus (IR) muscles, respectively (Figure 1a \&1b).

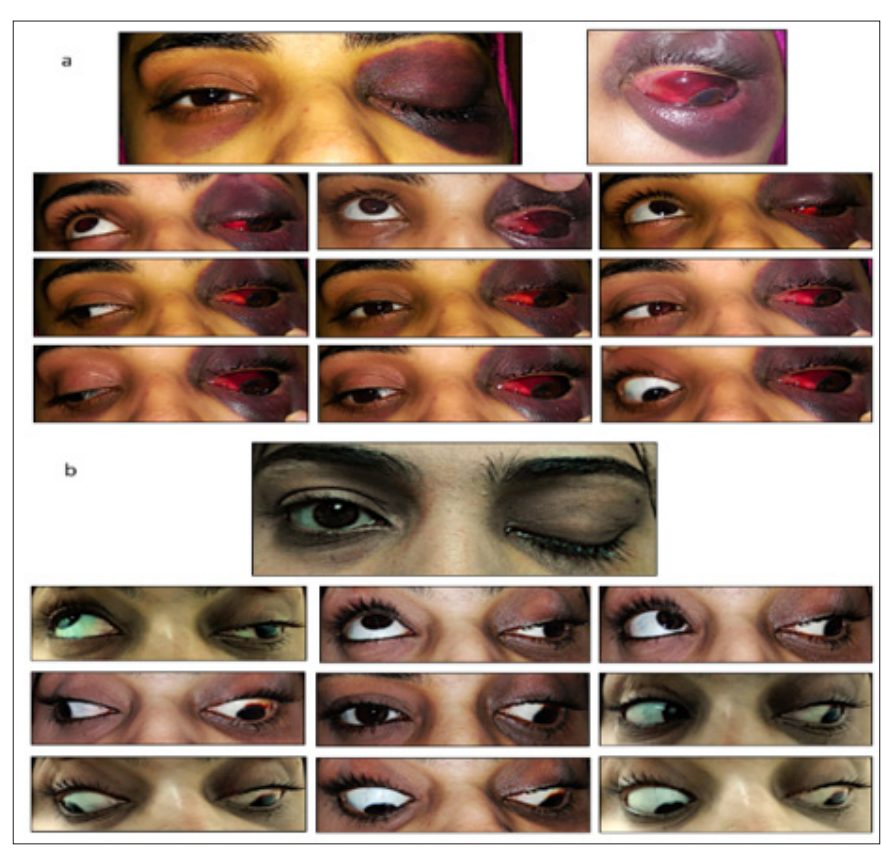

Figure 1: a) 3 days after Functional Endoscopic Sinus Surgery (FESS); b)8 weeks after FESS.

CT scan (Figure 2a) and MRI (Figure 2b) of the orbits, sinuses, and brain were performed. These showed, a large upper nasal septal defect and defective posterior $1 / 3$ of left lamina papyracea. The left MR muscle and optic nerve were entrapped within the lamina papyracea defect with extra-orbital herniation of the intraorbital fat into the ethmoidal air cells. The sharp edge of the lamina papyracea defect was embedded within the optic nerve. The left MR and optic nerve were not seen continuous in any cut (injured versus masked by orbital fat). The origins of left SO and MR muscles were swollen (contusion). The muscle bellies of left extraocular muscles along the anterior $1 / 3$ of the orbit were swollen. The right orbit was normal. The left maxillary sinus showed an antrostomy, with polypoidal thickening and retained secretions. The right maxillary antrum showed high density fluid that could be hemorrhagic. There was frontal and ethmoidal sinusitis, with rarefaction of the bony ethmoidal septa.

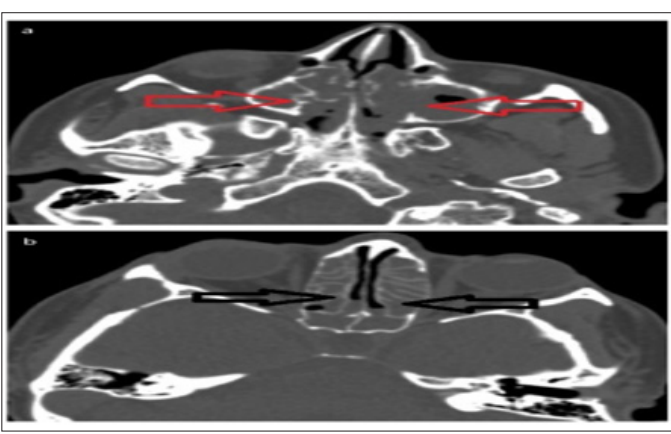

Figure 2: Pre-operative non-contrast CT scan of the paranasal sinuses and orbit, axial cuts, bone window, (a) showing opacified both maxillary antra (red arrows), and (b) showing opacified ethmoidal air cells on both sides (black arrows) and rarified, yet intact, bony septae. 
After 8-weeks' follow-up, the clinical picture was essentially the same, but with resolution of ecchymosis (Figure 1b) and development of an epiretinal membrane (Figure 3). The diagnosis of left optic nerve injury, CRAO, MR entrapment and palsy of the superior division of the oculomotor nerve was presumed. Ischemia was assumed as the cause with resultant orbital infarction syndrome. Seven months after FESS, there was no improvement of ocular motility and alignment. Orbital surgery was performed. Forced duction test of MR and LR was positive. The medial orbital wall fracture was exposed through a transcarancular medial orbitotomy. The optic nerve and MR were found entrapped. The
MR was freed. Medpore implant was used to close the medial orbital wall defect. Two weeks postoperatively, there was hardly any improvement in ocular motility or alignment and the picture remained essentially the same. Ten months after FESS, the patient underwent strabismus surgery. Forced duction test was positive, with extensive adhesions, but intact MR muscle. Surgery in the form of left LR recession $9 \mathrm{~mm}$ and left MR resection $6.5 \mathrm{~mm}$ was done. There was marked improvement of ocular alignment, motility and ptosis. Six weeks postoperatively, she had a residual left hypotropia 15 PD and XT 30 PD, with limited elevation and adduction, and partial ptosis (Figure 4).
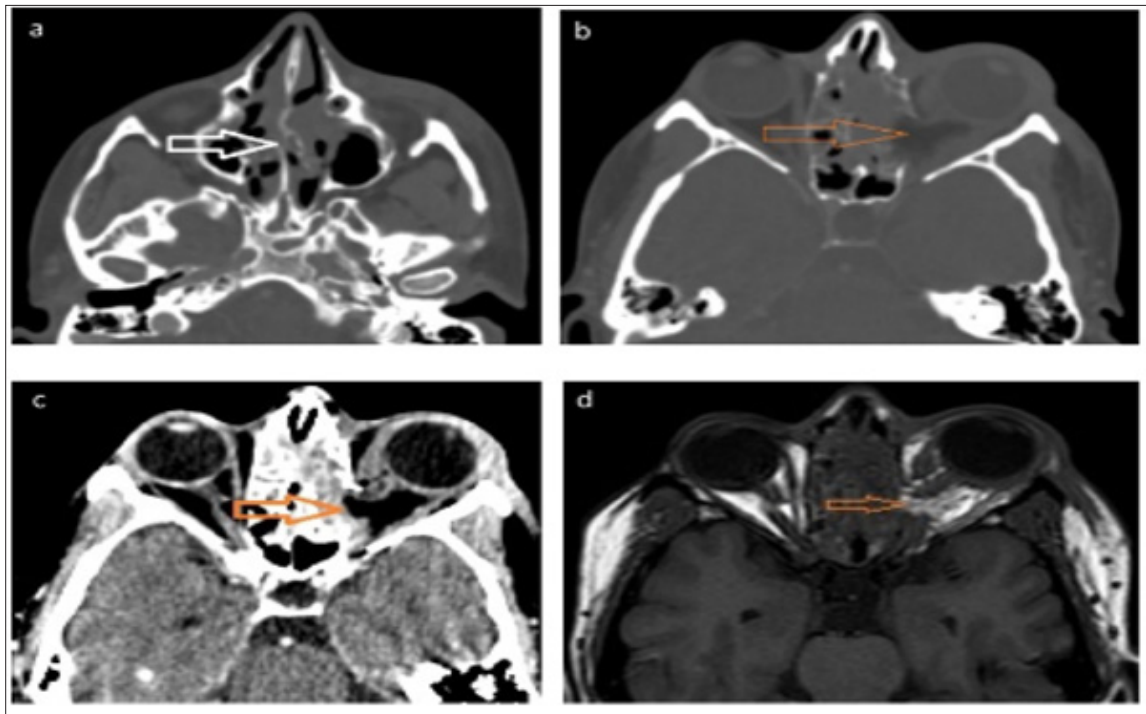

Figure 3: Post-operative imaging $(\mathrm{a}, \mathrm{b}, \mathrm{c})$ non-contrast CT scan of the paranasal sinuses and orbit, axial cuts, (d) Non-contrast MRI T1WIs of the paranasal sinuses and orbit, axial cuts, showing defect in the bony nasal septum (white arrow), destruction of the inter-ethmoidal septae on the left side, defect in the medial orbital wall, loss of the continuity of the medial rectus muscle, loss of the continuity of the left optic nerve and its entrapment medially at the defect of the medial orbital wall (orange arrows), with blurring of the retro-orbital fat in MRI image.

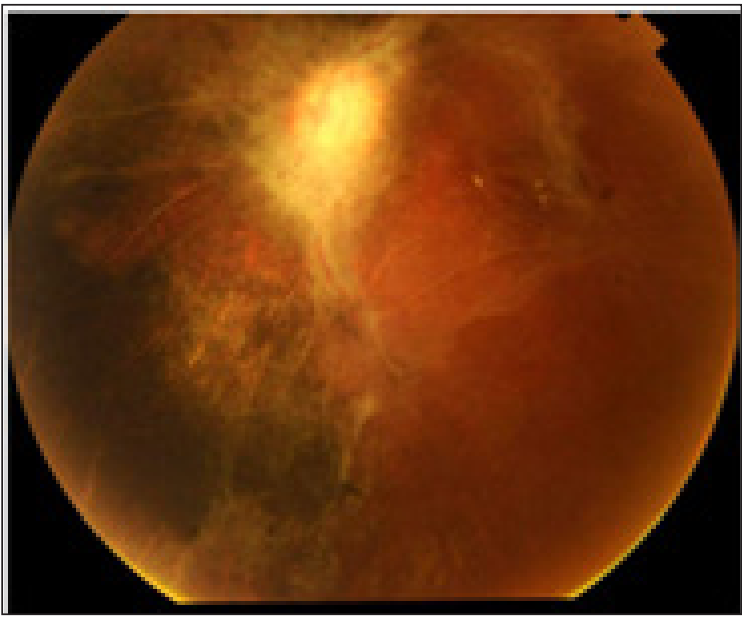

Figure 4: Fundus picture 8 weeks after FESS. Attenuated retinal arteries, subretinal pigmentation, and epiretinal membrane over the posterior pole.

\section{Discussion}

The exact details of what happened during FESS remained obscure. Possible scenarios are presumed. The marked widening of the minute nasal septal defect (detected in a CT scan performed prior to the third FESS, Figure 5 suggests gross injury of the nasal septum by the surgical instruments. The latter then crossed from the right to left side, through the septal defect, to injure the left lamina papyraea, where rarefaction of the ethmoid sinus facilitated entry into the left orbit. MR entrapment, followed by optic nerve entrapment, into the lamina papyracea defect ensued. Trauma to the optic nerve probably caused spasm of the Central Retinal Artery (CRA), with subsequent occlusion. Alternatively, the CRA was injured rather than mere spasm. Lastly, the instruments injured the superior division of oculomotor neve that crosses superiorly on the lateral aspect of the optic nerve to supply the SR and Levator Palpebrae Superioris (LPS) muscles. Alternatively, limited ocular motility was due to extraocular muscles contusion rather than oculomotor nerve injury. Limited motility might be also caused by injury of ophthalmic artery, with resultant ischemia of extraocular muscles. 


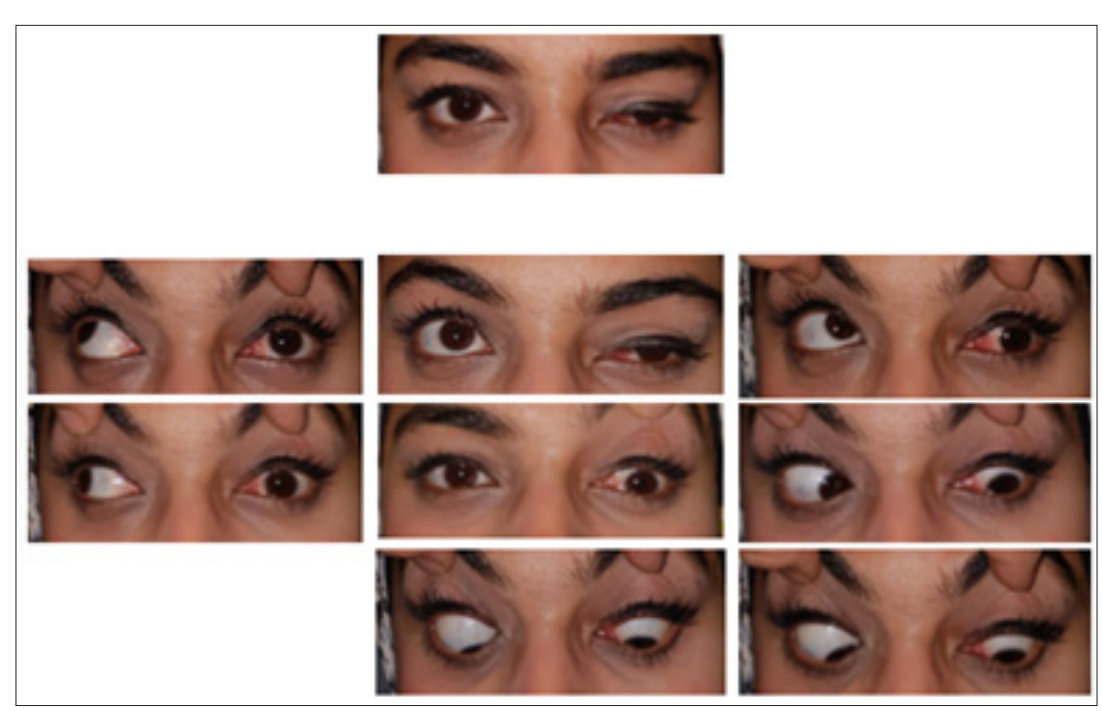

Figure 5

The inferior division of the oculomotor nerve was intact, evidenced by preserved IR function as well as preserved IO function (evidenced by extorsion on attempted elevation in adduction). The limited adduction could be attributed to entrapment of the MR rather than injury of the inferior division of oculomotor nerve. The trochlear nerve was also intact as the SO could intort the globe on attempted depression in adduction, yet the SO muscle might have been partially injured, as detected on MRI. The nasociliary nerve was intact since corneal sensation was preserved. Acute postoperative vision loss is a rare but devastating complication of FESS. Loss of vision might be caused by orbital hemorrhage with optic nerve compression, or direct optic nerve trauma, or ischemic optic neuropathy [2].Optic nerve injury is suspected if the pupil rapidly dilates during surgery or if, after surgery, there is severe visual loss and relative afferent pupillary defect.1 Other causes of vision loss after FESS include: injury to orbital structures, thromboembolic events, retinal migraine, and ophthalmic artery vasospasm. Rapid identification of the underlying etiology of vision loss is paramount because permanent vision loss often occurs in 60-90 minutes following vascular compromise and treatment differs according to the etiology [3].

Orbital hematoma is the most feared orbital complication following sinus surgery is. Orbital hematoma is recognized by rapidly increasing proptosis, subconjunctival hemorrhage, and perorbital ecchymosis. Severe orbital hemorrhage causes compressive optic neuropathy. This can be treated by orbital decompression and evacuation of the hematoma [4]. "Orbital Compartment Syndrome" was described with small or large orbital hematomas, causing rapid rise of intraorbital pressure, with resultant CRAO [5]. In our case, mild proptosis, subconjunctival hemorrhage and lid ecchymosis were noted on the second postoperative day. Orbital hemorrhage was probable. Ophthalmic artery vasospasm was repeatedly documented as a cause of acute visual loss after FESS. The anterior and posterior ethmoidal arteries provide a direct connection of the nasal cavity and orbit via their continuity with the ophthalmic artery. There are several documented cases of ophthalmic artery vasospasm following FESS thought to be secondary injection of local anesthetic with epinephrine within the nasal cavity or direct injection of epinephrine into the ethmoidal artery [6]. In our case, general anesthesia was used, so epinephrine injection was unlikely. Byrd et al. reported a case of acute vision loss following FESS due to suspected ophthalmic artery vasospasm in absence of orbital hematoma or local injection [3]. Standard therapy for vasospasm includes prompt treatment with calcium channel blockers [7]. Byrd et al. successfully treated their case with sublingual nitroglycerin [3].The presumed cause of CRAO in our case is CRA vasospasm secondary to optic nerve trauma. Ipsilateral permanent visual loss ensued.

The optic nerve was incarcerated in the lamina papyracea defect and the sharp edge of the latter was embedded within the optic nerve in our case. Similarly, Rene et al. reported a case where on CT scanning, following endoscopic sinus surgery, the middle portion of the lamina papyracea was defective, the optic nerve was adherent to the defect and one or two small specks of bone density were seen in the vicinity of the optic nerve.8

The left MR was entrapped in the defect in the lamina papyracea in our study. A similar condition was reported by Rene and coauthors. Although in their case the tissues were freed and the orbital wall was repaired by a silicone sheet, exotropia persisted and necessitated a LR recession [8]. Similarly, exotropia persisted in our case despite setting the entrapped MR free. Oculomotor nerve palsy after FESS is extremely rare. Rao et al. reported a case of CRAO and oculomotor nerve palsy following nasal septoplasty in a patient presenting with epistaxis [9].

This differed from our case where FESS and not nasal septoplasty was performed. Later Kim et al. reported the first case of oculomotor nerve palsy (ptosis, limited adduction, supraduction, and infraduction) following FESS for nasal polyposis. The patient had normal anterior and posterior segments. CT scan revealed 
dehiscence of the lamina papyracea posteriorly, with protrusion of soft tissue. Full recovery ensued after systemic dexamethasone for 2 weeks. The authors attributed the cause to focal nerve contusion or ischemic nerve injury [10]. This differed from our case, where infraduction was normal and there was trauma to the optic nerve, CRAO, and vision loss. Only mild improvement of ocular motility was detected after up to 8-weeks follow-up. "Orbital Infarction Syndrome" was previously described after surgery for intracranial aneurysms. There is global orbital infarction resulting from ischemia of intraocular and intraorbital structures due to hypoperfusion of the ophthalmic artery. These patients develop acute proptosis, ophthalmoplegia, and blindness [11]. This clinical picture mimics our case. Accordingly, the diagnosis of orbital infarction syndrome is presumed here.

\section{Conclusion}

This case report presents the first combination of optic nerve injury, CRAO, together with superior division oculomotor nerve palsy following FESS. It also presents the first case of intra orbital injury contralateral to the side of FESS. Care should be taken during FESS to avoid intra orbital injury. More care is essential in case of pre-existing nasal septal defect or rarefaction of ethmoid sinus to avoid contralateral orbital complications.

\section{References}

1. Bhatti MT (2007) Neuro-ophthalmic complications of endoscopic sinus surgery. Curr Opin Ophthalmol 18(6): 450-458.

ISSN: 2574-1241

DOI: 10.26717/BJSTR.2019.13.002470

Rehab R Kassem. Biomed J Sci \& Tech Res

(C) This work is licensed under Creative

Submission Link: https://biomedres.us/submit-manuscript.php
2. Pelausa EO, Smith K, Dempsey I (1995) Orbital complications of functional endoscopic sinus surgery. J Otolaryngol 24(3): 154-159.

3. Byrd S, Hussaini AS, Antisdel J (2017) Acute vision loss following endoscopic sinus surgery. Case Reports in Otolaryngology 2017: 3.

4. Stankiewicz JA (1989) Blindness and intranasal endoscopic ethmoidectomy: Prevention and management. Otolaryngol Head Neck Surg 101(3): 320-329.

5. Klenk G, Katona J, Kenderfi G, Lestyan J, Gombos K, et al. (2017) Orbital compartment syndrome. The most frequent cause of blindness following facial trauma. Orv Hetil 158(36): 1410-1420.

6. Savino PJ, Burde RM, Mills RP (1990) Visual loss following intranasal anesthetic injection. Journal of Clinical Neuro-Ophthalmolgy 10(2): 140144 .

7. Flammer J, Pache M, Resink T, Vasospasm (2001) Its role in the pathogenesis of diseases with particular reference to the eye. Progress in Retinal and Eye Research 20:319-349.

8. Rene C, Rose GE, Lenthall R, Moseley I (2001) Major orbital complications of endoscopic sinus surgery. Br J Ophthalmol 85(5): 598-603.

9. Rao GN, Rout K, Pal A (2012) Central retinal artery occlusion and third cranial nerve palsy following nasal septoplasty. Case Rep Ophthalmol 3(3): 321-326.

10. Kim TS, Lee JH, Kim M (2016) Transient oculomotor nerve palsy after endoscopic sinus surgery. Indian J Ophthalmol 64(9): 697-698.

11. Zimmerman CF, Van Patten PD, Golnik KC, Kopitnik TA Jr, Anand R (1995) Orbital infarction syndrome after surgery for intracranial aneurysms. Ophthalmology 102(4): 594-598.

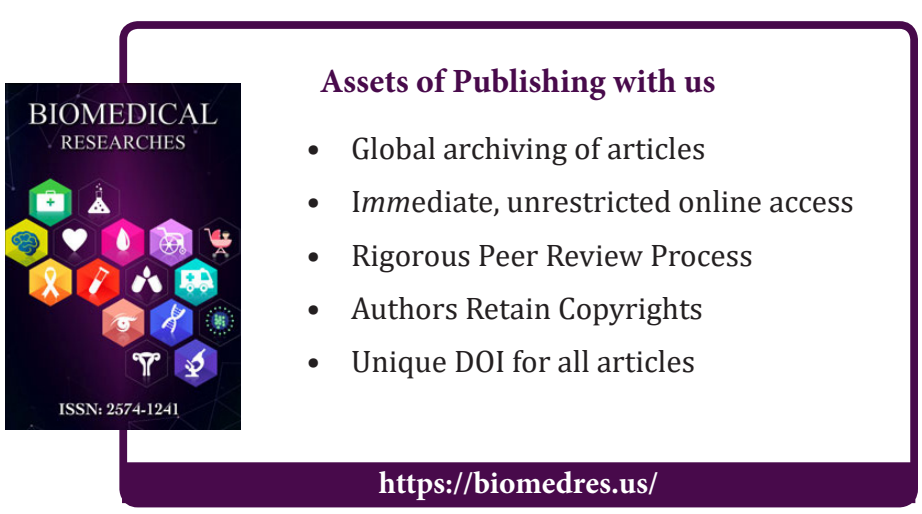

